\title{
Impacts of torus model on studies of geometrical relationships between interplanetary magnetic clouds and their solar origins
}

\author{
Katsuhide Marubashi ${ }^{1}$, Suk-Kyung Sung ${ }^{1}$, Kyung-Suk Cho ${ }^{1}$, and Ronald P. Lepping ${ }^{2}$ \\ ${ }^{1}$ Space Science Division, Korea Astronomy and Space Science Institute, Daejeon 305-348, Korea \\ ${ }^{2}$ Space Weather Laboratory, NASA-Goddard Space Flight Center, Greenbelt, MD 20771, USA
}

(Received December 14, 2007; Revised April 17, 2008; Accepted April 18, 2008; Online published May 29, 2009)

\begin{abstract}
Our recent analysis of interplanetary magnetic clouds (MCs) showed that the orientations of MC axes determined by a model fitting with curvature of MCs taken into account (referred to as a torus model, hereafter) can be significantly different from those obtained from fittings with a straight cylinder model. Motivated by this finding, we re-examined geometrical relationships between magnetic field structures of MCs and their solar origins. This paper describes the results of the re-examination with special attention paid to two MC events, for which different orientations of MC axes were obtained from a torus model and a cylinder model. For both cases, it is shown that the torus models give the MC geometries of magnetic field structures in good agreement with those of coronal arcade structures which were formed in association with the launch of MCs along the magnetic field inversion lines. Summarizing the analysis results for $12 \mathrm{MCs}$ investigated here, we conclude that: (1) the formation of coronal arcade structure is a good indication of MC formation; (2) MC geometries can be obtained that are consistent with the coronal arcades with respect to the axis orientation and the magnetic field structure including chirality, indicating that no significant direction changes occurred during the propagation of MCs through the interplanetary medium.
\end{abstract}

Key words: Interplanetary magnetic clouds, magnetic field structures, solar origins, geometrical relationships.

\section{Introduction}

The interplanetary magnetic cloud (MC) occupies the whole body or a significant part of a plasma cloud ejected into the solar wind in association with the coronal mass ejection (CME). The internal structure of a MC is characterized by the nested helical fields, which can be described as a magnetic flux rope (Goldstein, 1983; Marubashi, 1986; Burlaga, 1988). The geometrical relationship between magnetic structures of MCs and their solar source region is one of the key issues for understanding the mechanisms involved in the formation of MCs and their possible deformation during their propagation in interplanetary space. Most previous studies on this subject have shown that the orientations of MCs are roughly aligned with the inversion lines of photospheric longitudinal magnetic fields in the associated CME sites and that the chirality of MCs are of the same sign as the chirality of solar magnetic fields in the source regions (e.g., Marubashi, 1986, 1997; Bothmer and Schwenn, 1994; Bothmer and Rust, 1997; Yurchyshyn et al., 2001). More recent studies, however, point out several cases in which the orientations of MCs do not agree with those of the associated filaments or solar magnetic inversion lines (Rust et al., 2005; Wang et al., 2006; Yurchyshyn et al., 2007).

The purpose of this study is to re-examine the geometrical relationships between the MC magnetic fields and the solar magnetic fields with a new technique to determine

Copyright (c) The Society of Geomagnetism and Earth, Planetary and Space Sciences (SGEPSS); The Seismological Society of Japan; The Volcanological Society of Japan; The Geodetic Society of Japan; The Japanese Society for Planetary Sciences; TERRAPUB. the orientations of MCs. In most studies on this subject, as mentioned above, the MC orientations were determined by fitting techniques with a straight cylinder model. Recently, Marubashi and Lepping (2007) analyzed the MC geometries using a torus-shaped flux rope model to take into account the curvature of the global configuration of MCs. Their comparison of the MC orientations determined from the torus and cylinder models revealed that there actually are such MC events for which observations can be as well explained by either of the models but that the obtained orientations are significantly different from each other. This finding impacts on our understanding the connection between MCs and CMEs and suggests that it may be possible to obtain a better agreement between the MC orientations and the solar magnetic inversion lines if the MC geometries are determined with a torus model.

The MC database for this study consists of the analysis results of 17 long-duration MCs (Marubashi and Lepping, 2007). We compared the MC geometries obtained from the torus and cylinder models with the magnetic field structures in the possible solar source regions of the MCs. We present here our analysis results, with a focus on two events for which the connection between the MC and related solar phenomena can be seen in a straightforward way. Similar connections are also seen for other MC events.

\section{Analysis Results}

Figure 1 shows the fitting results of the $\mathrm{MC}$ encountered on March 4, 1998, with solid lines for the torus model and dotted lines for the cylinder model, superposed on the ob- 


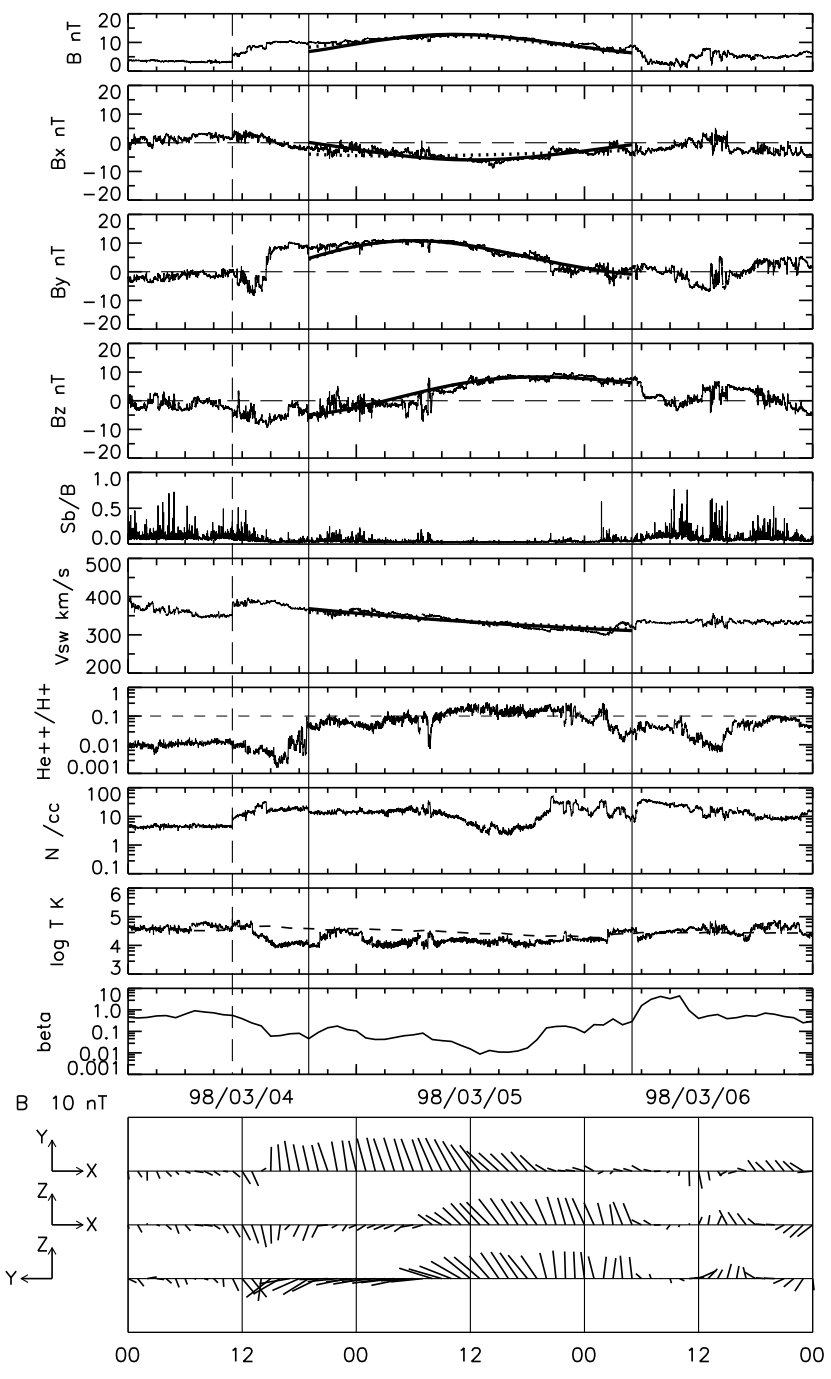

Fig. 1. Results of fitting the torus model (solid curve) and the cylinder model (dotted curve) to the magnetic cloud encountered on March 4, 1998. The bottom three panels show the projected magnetic field vectors onto three planes in GSE coordinates.

served variations of solar wind magnetic fields during a 3day interval. Plotted from top to bottom are the magnetic field intensity, the $X, Y$, and $Z$ components in GSE (geocentric solar ecliptic) coordinates, the ratio of standard deviations of high-resolution data to the average intensity, the solar wind speed, the number density ratio of $\mathrm{He}^{++} / \mathrm{H}^{+}$, the proton number density, the proton temperature, the plasma beta based on protons, and the magnetic field vectors projected on the $X-Y, X-Z$, and $Y-Z$ planes of GSE coordinates. The dashed curve drawn along with the proton temperature shows the temperature statistically expected from the solar wind speed (Lopez, 1987). Two vertical solid lines indicate the MC boundaries as determined by such characteristics as the magnetic field rotation, the enhanced $\mathrm{He}^{++} / \mathrm{H}^{+}$ratio, the relatively small magnetic field fluctuations, and the abnormally low proton temperature (Richardson and Cane, 1993). The vertical dashed line indicates the shock associated with this MC. The observed variations in magnetic field and solar wind speed are well reproduced by both the torus and cylinder models, so that the difference between the two fitted curves can be hardly distinguished in

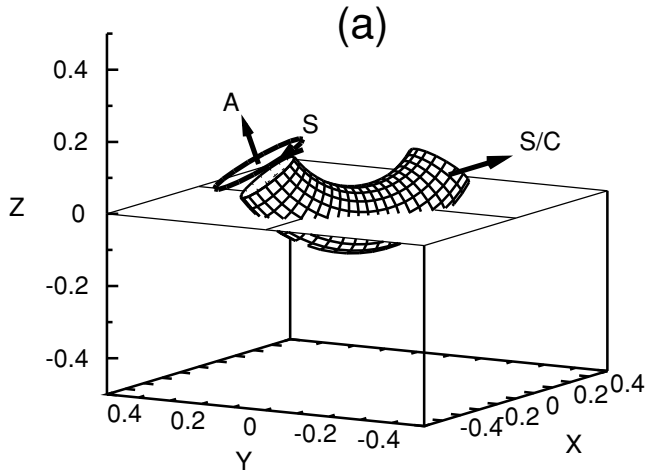

(b)

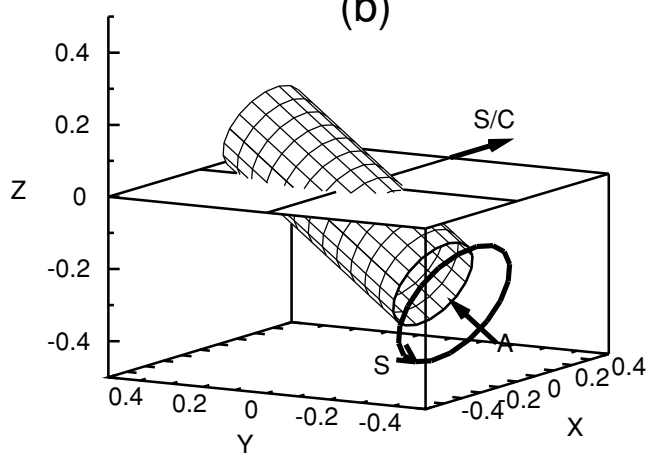

Fig. 2. Two geometries obtained for the magnetic cloud of March 4, 1998, from the fittings to the torus model (a) and to the cylinder model (b). Three directions are indicated: A, axial field; S, toroidal field on the surface; $\mathrm{S} / \mathrm{C}$, spacecraft trajectory relative to the magnetic cloud.

this presentation. However, the MC orientations obtained from the two models are substantially different from each other.

Figure 2 depicts the MC geometry obtained from the torus model (Fig. 2(a)) and that from the cylinder model (Fig. 2(b)), in which three arrows indicate the direction of the axial field of MC (A), the direction of magnetic field on the MC surface (S), and the direction of spacecraft trajectory $(\mathrm{S} / \mathrm{C})$. We can see that this MC has the lefthanded magnetic chirality from the two arrows, A and S. At the location where the spacecraft traversed the MC, the approximate direction of the torus axial field is given by $\theta$ (latitude angle $)=17.4^{\circ}$ and $\phi$ (longitudinal angle) $=$ $136.9^{\circ}$, whereas the direction of the cylinder axis is given by $\theta=29.3^{\circ}$, and $\phi=76.7^{\circ}$ (for details of the fitted values, including other parameters, see Marubashi and Lepping, 2007).

In an attempt to identify the most plausible solar source event of this MC, we searched the LASCO CME cata$\log$ (Yashiro et al., 2004; see also the website http://cdaw. gsfc.nasa.gov/CME_list) for candidate CMEs and related solar phenomena within a selected time window. For this purpose, we first estimate the launch time of the CME corresponding to this $\mathrm{MC}$, assuming that the MC propagated at a constant speed. In accordance with three values for the typical speeds, $340 \mathrm{~km} / \mathrm{s}$ (from the cylinder fitting), $350 \mathrm{~km} / \mathrm{s}$ (from the torus fitting), and $365 \mathrm{~km} / \mathrm{s}$ (the maximum speed within the MC), the launch time is estimated to be in the interval from 15:00 UT, February 27 to 01:00 UT, February 28, 1998. Thus, we select a time window from 03:00 UT, 
(a)

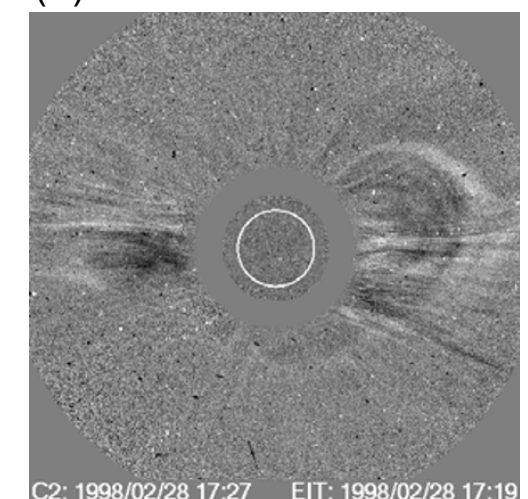

(c)

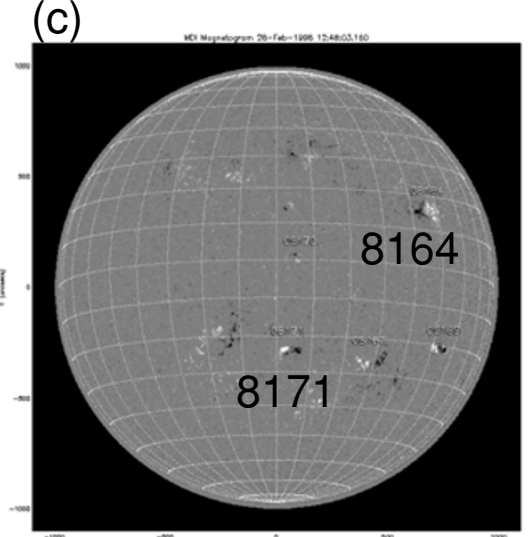

(b)

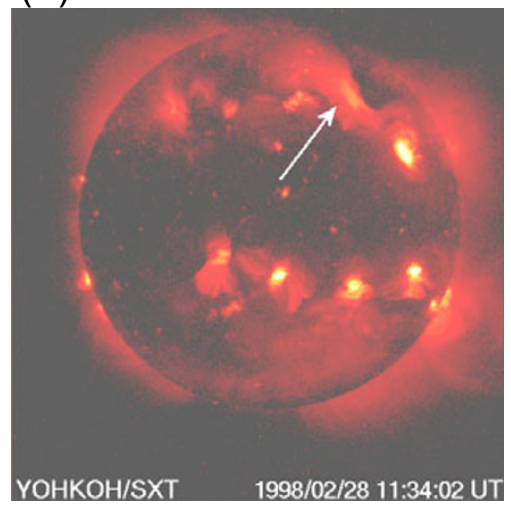

(d)

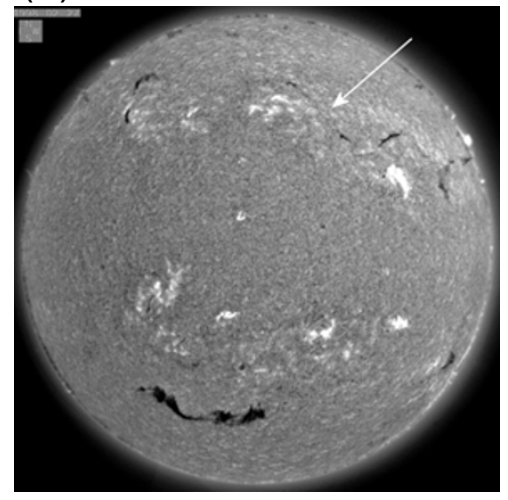

(e)

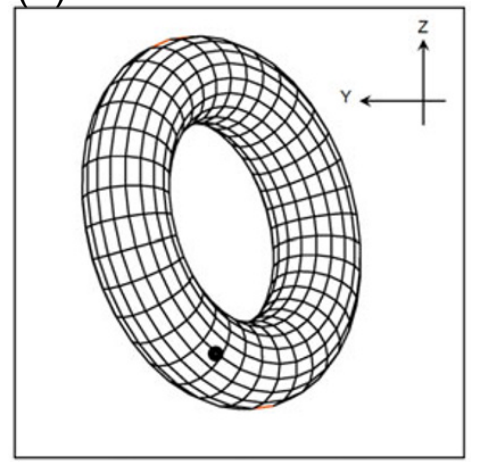

Fig. 3. Solar observations related to the March 4, 1998 MC: (a) LASCO C2 CME running difference image at 17:27 UT, February 28, 1998; (b) Yohkoh/SXT image at 11:34 UT; (c) SOHO/MDI image 12:48 UT, (d) BBSO H $\alpha$ image at 19:15 UT, February 27; (e) the torus MC inclination inferred from a local fit with a torus model.

February 27 to 13:00 UT, February 28, allowing an extra $\pm 12 \mathrm{~h}$ from the estimated launch time for searching the CME corresponding to the MC. The CME catalog shows that there were two CMEs with an angular width larger than $120^{\circ}$ in this time window. The first one is a full halo CME which appeared in the LASCO C2 field of view first at 20:07 UT on February 27, 1998, and the second one is a partial halo CME with angular width of $169^{\circ}$ that appeared at 12:48 UT on February 28, 1998. Of these two, the latter was identified as a solar source event of the March 4, 1998 MC by Gopalswamy et al. $(2000,2001)$, the speed of which is estimated to be approximately $300 \mathrm{~km} / \mathrm{s}$ at $20 \mathrm{Rs}$. We adopt this selection and search for the solar source region of the CME for the purpose of comparing structures between the MC and solar magnetic fields.

Figure 3 shows images of solar observations relevant to generation of the MC of March 4, 1998 (a: SOHO/LASCO C2 image; b: Yohkoh/SXT, c: SOHO/MDI; d: BBSO H $\alpha$ ). The torus shape corresponding to the $\mathrm{MC}$ is also shown in Fig. 3(e) for the purpose of indicating the tilting angle of the fitted model. (It should be noted that the actual MC shape is just locally approximated by the torus.) During several hours before this CME (Fig. 2(a)) first appeared in the LASCO C2, we can see two kinds of prominent activities in the Yohkoh/SXT movie. One consists of small activities repeatedly observed in the AR 8171 (approx. $24^{\circ} \mathrm{S}$, approx. $1^{\circ} \mathrm{W}$ ), and the other is the formation of a soft X-ray arcade possibly associated with a filament eruption (Figs. 3(b, d)) along the neutral line extending from the north of AR 8164 towards the north-east in the region of weaker magnetic fields (see Figs. 3(c, d)), similar to the arcade formation exemplified by Tripathi et al. (2004). Although it is 


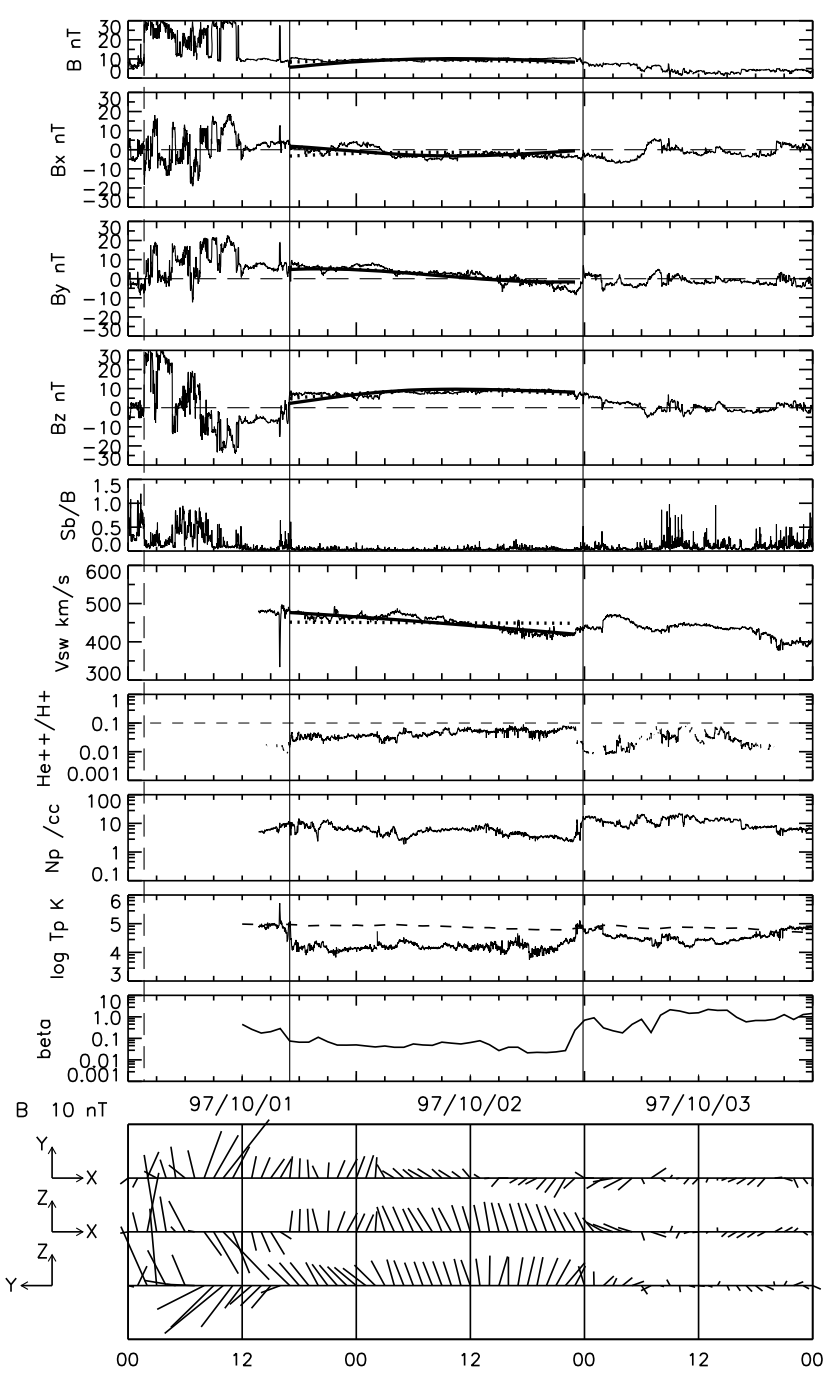

Fig. 4. Results of fitting the torus model (solid curve) and the cylinder model (dotted curve), respectively, to the magnetic cloud encountered on October 1, 1997. The bottom three panels show the projected magnetic field vectors.

difficult to rule out the association between the CME and some small activity in AR 8171, the arcade-formation event seems to be directly connected with the CME in Fig. 3(a). The latter association is more plausible based on several supporting pieces of evidence: (1) the orientation of the MC axis is close to the orientation of the arcade, as is evident by comparing Figs. 3(b, e); (2) the polarity of the magnetic field on the surface of the MC is consistent with the arcade magnetic field, as inferred from the MDI data; (3) the left-handed magnetic field chirality of MC agrees with the chirality of the coronal magnetic field expected from the inverse-S type sigmoid in this region (Rust and $\mathrm{Ku}-$ mar, 1996); (4) The chirality agrees with general tendency that the MCs with left-handed (right-handed) chirality are formed in association with the solar events in the northern (southern) hemisphere (Marubashi, 1986, 1997; Bothmer and Schwenn, 1994; Bothmer and Rust, 1997). Finally, it is possible to suppose from Fig. 3(a) that this CME was launched a little toward south, and that the ACE spacecraft passed the southern end of the MC loop as indicated by the dark dot in Fig. 3(e). (a)

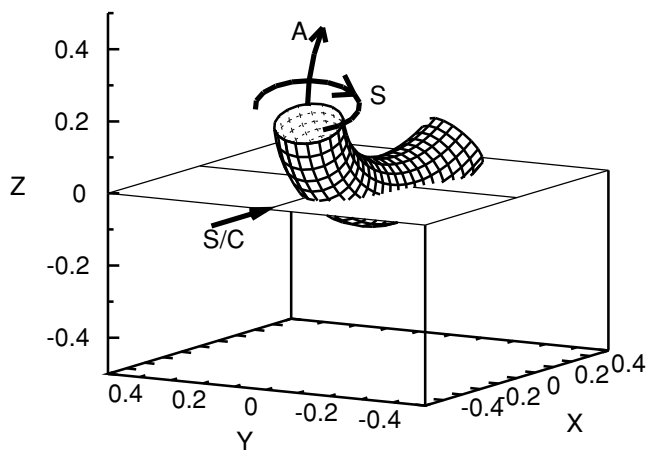

(b)

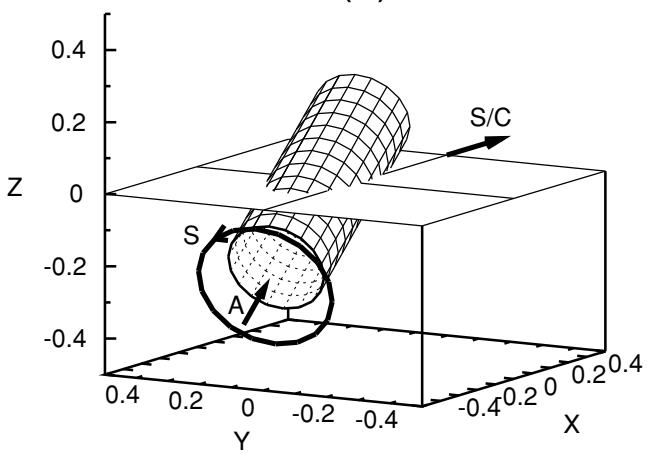

Fig. 5. Two geometries obtained for the magnetic cloud of October 1, 1997, from the fittings to the torus model (a) and to the cylinder model. Three directions are indicated: A, axial field; S, toroidal field on the surface; S/C, spacecraft trajectory relative to the magnetic cloud.

Figures 4 and 5 show the fitting results for another MC which was encountered on October 1, 1997, in the same format as Figs. 1 and 2. Again, both cylinder and torus models having the left-handed chirality reproduce the magnetic field variations agreeing well with the observation. It should be noted here that the torus parameters used here are different from original values presented in Marubashi and Lepping (2007). The orientation of the torus plane determined by the standard fitting method is given by $\theta_{\mathrm{n}}=35.1^{\circ}$, and $\phi_{\mathrm{n}}=65.0^{\circ}$, the latitude and longitude angles of a vector normal to the torus plane. This torus plane is tilted too much compared with the direction of the post-eruption arcade as will be seen later. Since our purpose is to examine a possibility to obtain the orientation of the $\mathrm{MC}$ axis which is close to the orientation of the inversion line of solar magnetic fields, we executed a least-squares method slightly modified from that applied in the previous work. We tried fitting with fixed $\phi_{\mathrm{n}}$, using different values around $\phi_{\mathrm{n}}=65.0^{\circ}$, and searched the parameter set which can reproduce the observed magnetic fields, and give geometries having the axis orientation as parallel to the inversion line as possible. Thus, we obtain a result shown in Fig. 4 for $\theta_{\mathrm{n}}=14.6^{\circ}$, and $\phi_{\mathrm{n}}=77.5^{\circ}$. The point is that we can get such an MC geometry by using a torus model.

As for the solar source of this MC, the CME catalog shows that there was only one CME with angular width greater than $120^{\circ}$ within the time window from 08:00 UT on September 27 to 14:00 UT on September 28, 1997, that is, the halo CME that appeared in the LASCO C2 field of 
(a)

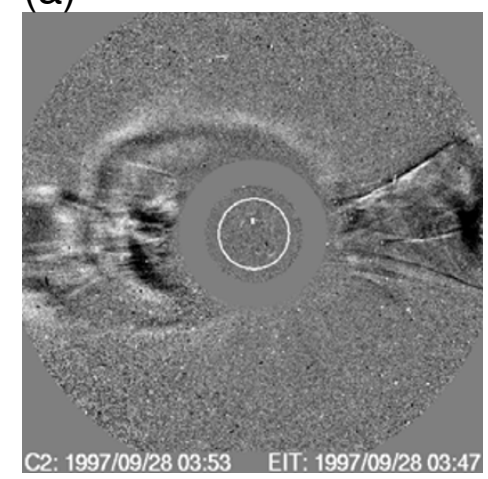

(c)

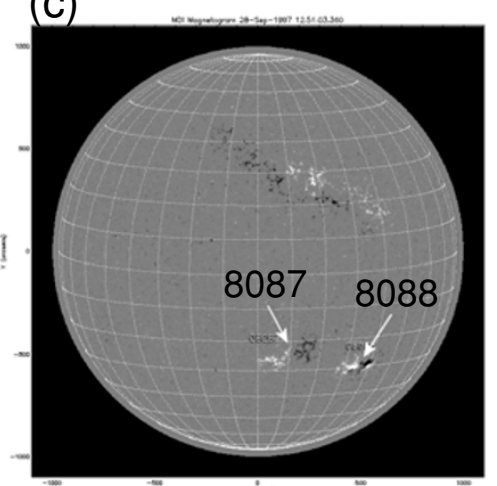

(e) (b)

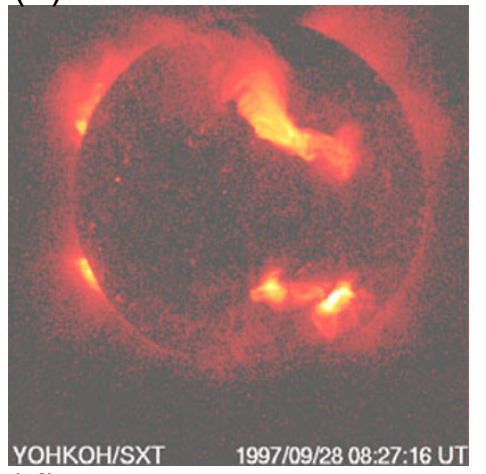

(d)

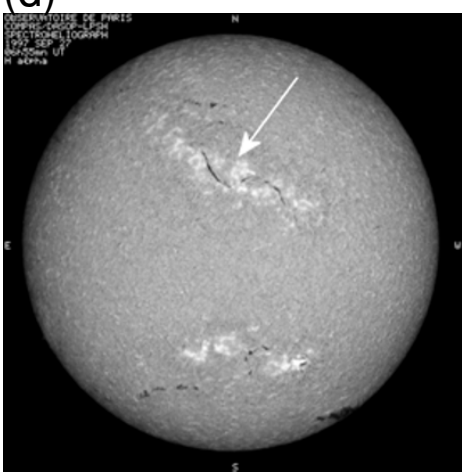

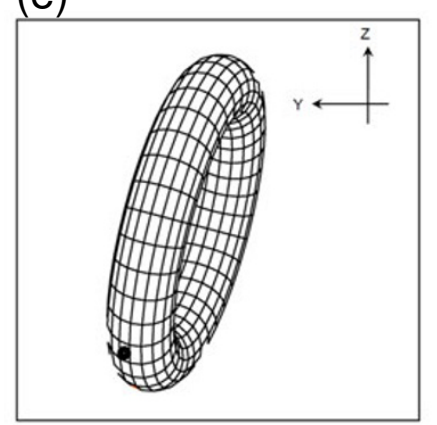

Fig. 6. Solar observations related to the October 1, 1997 MC: (a) LASCO C2 CME running difference image at 03:53 UT, September 28, 1997; (b) Yohkoh/SXT image at 08:27 UT; (c) SOHO/MDI image 12:51 UT, (d) Meudon H $\alpha$ image at 06:55 UT, September 27; (e) the torus MC inclination inferred from a local fit with a torus model.

view at 01:08 UT on September 28, 1997. Though there is a gap in the Yohkoh/SXT data from 22:39 UT September 27 to $08: 23$ UT September 28, we can see an arcade-formation activity which started around 22:12 UT on September 27 and developed rather slowly near the region surrounding the magnetic inversion line in the northern hemisphere. Gopalswamy et al. (2001) identified the solar source of the MC of October 1, 1977 to be this halo CME and associated it with the disappearing filament at $22^{\circ} \mathrm{N}, 05 \mathrm{E}$. Figure 6 presents the images relevant to the generation of the $\mathrm{MC}$, together with the torus tilt obtained from the fitting. Accepting this association, we see that the relationships between this interplanetary MC and the solar magnetic field are very much similar to the case of the March 4, 1998 MC. The MC with left-handed magnetic chirality was formed in association with the filament disappearance in the northern hemisphere, where an inverse-S type sigmoid had been observed. The orientation of the MC axis is nearly parallel to the neutral line or the erupted filament. The CME plasma was launched slightly southward rather than radially, and hit the Earth with its southern end, as seen in Figs. 5(a) and $6(\mathrm{e})$.

Thus far we have seen the analysis results for two MC events with special attention being paid to the geometrical relationships between MCs and the magnetic field structures in their possible solar source regions. Here, we briefly summarize results of our analysis of the 17 long-duration MCs. This analysis was carried out for only 12 of these MCs because solar observation data were insufficient for the other five MCs (for reference, we present the same event numbers used in Marubashi and Lepping, 2007; \#1, \#2, \#10, $\# 16$, and \#17). We were able to find plausible solar source event in six cases (\#4, \#5, \#7, \#12,\#13, and \#15), and for each of these, the magnetic inversion line on the Sun is consistent with the orientation of the MC axis determined by the torus fitting. We also found a formation of arcade-like structures along the magnetic inversion line within the solar source regions near the time of CME launch. For one 
case (\#14), no prominent solar activity was seen in the time window estimated by the MC observation. For the remaining five cases, more detailed analyses are needed both on the solar events and on the MC structures, although it is not impossible to find candidates as the solar sources.

\section{Conclusions and Discussion}

By analyzing the geometries of 12 long-duration MCs and their relationships with their solar origins, we have drawn the following conclusions.

(1) The arcade formation in the corona is a good indication of the generation of an MC.

(2) The magnetic field structure of a MC is consistent with the structure of a flux rope loop formed in the coronal region above the arcade, as various CME models commonly predict (see Forbes, 2000, for a review).

The above two findings are consistent with the view that MCs are expanding helical flux ropes formed in association with CME launch (Marubashi, 1997). The more comprehensive relation between the post-eruption arcades and flux rope structures in CMEs has been shown by Cremades and Bothmer (2004) and Tripathi et al. (2004).

(3) Furthermore, for MCs of which the orientations obtained from cylinder fitting mismatch the above relationship, it is possible to obtain an MC orientation which is consistent with the above by using a torus. This suggests that no significant direction change of MCs is needed during the propagation through the interplanetary medium.

It is desirable to extend this study further to other MC events of shorter durations because our study is focused only on the long-duration MCs. Such a study is currently underway, but we can point out that two MCs analyzed by Ishibashi and Marubashi (2004) and Crooker and Webb (2006), respectively, are good examples of where good relationships are obtained using a torus model to explain the alignment between the MC axis and the inversion line of coronal magnetic fields.

Acknowledgments. We gratefully acknowledge the use of data from the WIND SWE instrument, provided by the National Space Science Center at the Goddard Space Flight Center, and ACE MAG and SWEPAM instruments provided by the ACE Science Center (http://www.srl.caltech.edu/ACE/ASC/). We also acknowledge the use of solar data from the SOHO LASCO and MDI instruments, YOHKOH SXT instruments, and $\mathrm{H} \alpha$ images from Big Bear Solar Observatory and Meudon Observatory. We express our particular thanks to the many researchers involved in making and freeing providing the LASCO CME catalogs. This work was supported by the Development of Korean Space Weather Center, the project of KASI, and the KASI basic research fund.

\section{References}

Bothmer, V. and R. Schwenn, Eruptive prominences as sources of magnetic clouds in the solar wind, Space Sci.Rev., 70, 215-220, 1994.
Bothmer, V. and D. M. Rust, The field configuration of magnetic clouds and the solar cycle, in Coronal Mass Ejections, Geophys. Monogr. Ser., vol. 99, edited by N. Crooker, J. A. Joselyn, and J. Feynman, 139-146, AGU, Washington, D.C., 1997.

Burlaga, L. F., Magnetic clouds and force-free fields with constant alpha, J. Geophys. Res., 93, 7217-7224, 1988.

Cremades, H. and V. Bothmer, On the three-dimensional configuration of coronal mass ejections, Astron. Astrophys., 422, 307-322, 2004.

Crooker, N. U. and D. F. Webb, Remote sensing of the solar site of interchange reconnection associated with the May 1977 magnetic cloud, $J$. Geophys. Res., 111, A08108, doi:10.1029/2006JA011649, 2006.

Forbes, T. G., A review on the genesis of coronal mass ejections, J. Geophys. Res., 105, 23,153-23,165, 2000.

Goldstein, H., On the field configuration in magnetic clouds, in Solar Wind Five, edited by M. Neugebauer, 731-733, NASA Conf. Publ., CP-2280, 1983.

Gopalswamy, N., A. Lara, R. P. Lepping, M. L. Kaiser, D. Berdichevsky, and O. C. St. Cyr, Interplanetary acceleration of coronal mass ejections, Geophys. Res. Lett., 27, 145-148, 2000.

Gopalswamy, N., A. Lara, M. L. Kaiser, and J.-L. Bougeret, Near-Sun and near-earth manifestations of solar eruptions, J. Geophys. Res., 106, 25,261-25,277, 2001.

Ishibashi, H. and K. Marubashi, Structure of interplanetary magnetic cloud on April 16, 1999 and its origin estimated by fitting the torusshaped flux rope model, Geophys. Res. Lett., 31, L21807, doi:10. 1029/2004GL020702, 2004.

Lopez, R., Solar cycle invariance in solar wind proton temperature relationships, J. Geophys. Res., 92, 11,189-11,194, 1987.

Marubashi, K., Structure of interplanetary magnetic clouds and their solar origins, Adv. Space Res., 6(6), 335-338, 1986.

Marubashi, K., Interplanetary magnetic flux rope and solar filaments, in Coronal Mass Ejections, Geophys. Monogr. Ser., vol. 99, edited by N. Crooker, J. A. Joselyn, and J. Feynman, 147-156, AGU, Washington, D.C., 1997.

Marubashi, K. and R. P. Lepping, Long-duration magnetic clouds: a comparison of analyses using torus- and cylinder-shaped flux rope models, Ann. Geophys., 25, 2453-2477, 2007.

Richardson, I. G. and H. V. Cane, Signatures of shock drivers in the solar wind and their dependence on the solar source location, J. Geophys. Res., 98, 15,295-15,304, 1993.

Rust, D. M. and A. Kumar, Helical magnetic fields in filaments, Solar Phys., 155, 69-97, 1996.

Rust, D. M., B. J. Anderson, M. D. Andrews, M. H. Acuña, C. T. Russell, P. W. Schunk, and T. Mulligan, Comparison of interplanetary disturbances at the NEAR spacecraft with coronal mass ejections at the Sun, Astrophys. J., 621, 524-536, 2005.

Tripathi, D., V. Bothmer, and H. Cremades, The basic characteristics of EUV post-eruptive arcades and their role as tracers of coronal mass ejection source regions, Astron. Astrophys., 422, 337-349, 2004.

Wang, Y., G. Zhou, P. Ye, S. Wang, and J. Wang, A study of the orientation of interplanetary magnetic clouds and solar filaments, Astrophys. J., 651, 1245-1255, 2006.

Yashiro, S., N. Gopalswamy, G. Michalek, O. C. St. Cyr, S. P. Plunkett, N. B. Rich, and R. A. Howard, A catalog of white light coronal mass ejections observed by the SOHO spacecraft, J. Geophys. Res., 109. A07105, doi:10.1029/2003JA010282, 2004.

Yurchyshyn, V. B., H. Wang, P. R. Goode, and Y. Deng, Orientation of the magnetic fields in interplanetary flux ropes and solar filaments, Astrophys. J., 563, 381-388, 2001.

Yurchyshyn, V., Q. Hu, R. P. Lepping, B. J. Lynch, and J. Krall, Orientations of LASCO halo CMEs and their connection to the flux rope structure of interplanetary CMEs, Adv. Space Res., 40, doi:10. 1016/j.asr.2007.01.059, 2007.

K. Marubashi (e-mail: kmaru@kasi.re.kr), S.-K. Sung, K.-S. Cho, and R. P. Lepping 